\title{
908 HUMANIZED MODEL FOR ASSESSMENT OF THERAPIES TARGETING EITHER LYMPHOID OR MYELOID COMPARTMENT: ENHANCED EVALUATION OF CLINICAL RELEVANCY \& TRANSLATABILITY
}

${ }^{1}$ Florence Renart-Depontieu, 'Gaëlle Martin, ${ }^{1}$ Chloé Beuraud, ${ }^{2}$ Poonam Yakkundi, ${ }^{2}$ Angus Sinclair, ${ }^{3}$ Morgane Denis, ${ }^{3}$ Léa Magadoux, ${ }^{3}$ Elsa Kress, ${ }^{3}$ Charles Dumontet, ${ }^{4}$ Ludovic Bourre, ${ }^{4}$ Dean Campbell, ${ }^{4}$ Astrid Doerner, ${ }^{1}$ Patricia Isnard-Petit, ${ }^{1}$ Alexandre Fraichard, ${ }^{1}$ Yacine Cherifi, ${ }^{1}$ Kader Thiam*. 'genOway, Lyon, France; ${ }^{2}$ IGM Biosciences, Mountain View, CA, USA; ${ }^{3}$ Antinéo, Lyon, France; ${ }^{4}$ Crown Bioscience, Paris, France

Background The breakthrough of immunotherapies has unleashed new hope and new success for cancer therapy. However, the choice of a preclinical model is one of the main challenges as they are important for evaluation of translatability to help support testing in clinical studies, including potential efficacy and tolerability of immunotherapies during preclinical development.The development of mouse models featuring a human immune system (HIS) provides new paths for the investigation of the efficacy of immunotherapies in preclinical models engrafted with human tumors. Although these models provided a breakthrough in the assessment of immune targeting agents, they also come with a few significant caveats. These include: a lack of a mature human myeloid compartment in the mouse, and a short life span of the model when this compartment is promoted at non-physiological levels via the over-expression of human cytokines. Here, we report a novel mouse model (BRGSF-HIS), featuring functional human lymphoid and myeloid compartments. The human immune response of this model was assessed through examination of the immune cells composition in the tumor microenvironment (TME), and its ability to respond to biologics known to trigger cytokine release syndrome (CRS).

Methods BRGSF (balb/C Rag2-/-, IL2Rg-/-, SIRPaNOD and Flt3-/-) is a highly immunodeficient mouse, with reduced murine myeloid cells, which allows long term CD34+ HSCengraftment and development of human lymphoid and myeloid compartments (human CD141+ and CD1c+ DC subsets, CD123+ pDC, CD14+ monocytes), in blood, spleen and bone marrow. The engraftment is stable for over twelve months with no side effects. The effect of exogenous human Flt3 ligand (Flt3L) on the composition of TME in A549 model, and an anti-CD3 antibody (OKT3)-induced CRS, were assessed.

Results Exogenous human Flt3L significantly and transiently increased the proportion of human myeloid cells. This can be recalled by continuous dosing of Flt3L. Assessment of tumor immunobiology in A549 model, showed increased tumor-infiltrating T-cells (mainly CD8 + T-cells) and myeloid cells, while tumor-infiltrating NK cells were decreased. The presence of myeloid cells provides a new opportunity for assessment of myeloid targeted therapies, as proven using pDC-depleting antibodies. OKT3 administration resulted in CRS symptoms including a temperature drop, body weight loss and a change in serum cytokine levels. Symptoms were mitigated upon administration of tocilizumab, suggesting the contribution of the myeloid compartment in the response observed.

Conclusions These data demonstrate that BRGSF-HIS mice support development of functional human myeloid cells and that this mouse model enables preclinical evaluation of cancer immunotherapy in vivo.

http://dx.doi.org/10.1136/jitc-2021-SITC2021.908 